\title{
A Polymorphism rs6726395 in Nrf2 Contributes to the Development of Emphysema-Associated Age in Smokers Without COPD
}

\begin{abstract}
A. Sugitani, K. Asai, T. Watanabe, T. Suzumura, K. Kojima, H. Kubo, K. Sato, N. Ijiri, K. Yamada, T. Kimura, S. Fukumoto, K. Hirata, T. Kawaguchi
\end{abstract}

\begin{tabular}{|c|l|}
\hline Citation & Lung. 197(5); 559-564. \\
\hline Published & 2019-07-11 \\
\hline Type & Journal Article \\
\hline Textversion & Author \\
\hline & $\begin{array}{l}\text { This is a post-peer-review, pre-copyedit version of an article published in Lung. } \\
\text { The final authenticated version is available online at: } \\
\text { Rights }\end{array}$ \\
$\begin{array}{l}\text { https://doi.org/10.1007/s00408-019-00251-2. } \\
\text { See Springer Nature terms of use. } \\
\text { https://www.springer.com/gp/open-access/publication-policies/aam-terms-of-use. }\end{array}$ \\
\hline DOI & $10.1007 /$ s00408-019-00251-2 \\
\hline
\end{tabular}

Self-Archiving by Author(s)

Placed on: Osaka City University

Sugitani, A., Asai, K., Watanabe, T. et al. A Polymorphism rs6726395 in Nrf2 Contributes to the Development of Emphysema-Associated Age in Smokers Without COPD. Lung 197, 559-564 (2019). Doi: 10.1007/s00408-019-00251-2 
A polymorphism rs6726395 in Nrf2 contributes to the development of emphysema associated age in smokers without COPD

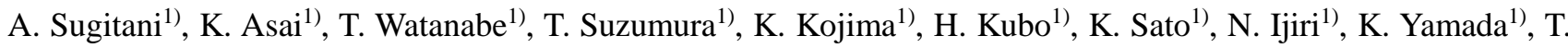

Kimura $^{2)}$, S. Fukumoto ${ }^{2)}$, K. Hirata ${ }^{1)}$, T. Kawaguchi ${ }^{1)}$

1) Department of Respiratory Medicine, Graduate School of Medicine, Osaka City University

2) Department of Premier Preventive Medicine, Graduate School of Medicine, Osaka City University

Word count for the body of the manuscript: 2421

Word count for the abstract: 250

Number of tables: 5

Number of figures: 4 


\section{Abstract}

Introduction

Several studies have reported that single nucleotide polymorphisms (SNPs) in the gene encoding NF-E2-related factor 2 (Nrf2) contribute to airflow limitations in smokers without COPD. Although small airway lesions and emphysema contribute cooperatively to airflow limitation, the relationship between Nrf2 SNPs and the development of emphysema in smokers without COPD is not well understood.

Methods

Healthy subjects who underwent an annual health checkup with computed tomography (CT) of the chest at Osaka City University Hospital were prospectively recruited. The percentage of low attenuation area (\%LAA) on chest CT was quantified and correlations between \%LAA, Nrf2 SNP [rs6726395 (G/A)] genotypes, and clinical characteristics were examined.

Results

A total of 245 subjects without COPD [non-/light-smoker: 153 (62.4\%) and smoker: 92 (37.6\%)] were enrolled. The \%LAA in the upper lung field was higher than that in the lower lung field $(\mathrm{p}<0.001)$. The \%LAA in smokers was significantly higher than in non-/light-smokers $(\mathrm{p}=0.021)$. The \%LAA showed significant but weak correlation with 
age in all subjects $(\mathrm{r}=0.141, \mathrm{p}=0.028)$. Divided by genotype, the \%LAA of the upper lung field was significantly correlated with age in smokers with genotype GG (wild type) $(\mathrm{r}=0.333, \mathrm{p}=0.022)$, but was not significantly correlated with age in smokers with genotype AG/AA. These correlations were not observed in non-/light smokers.

\section{Conclusion}

A polymorphism rs6726395 in Nrf2 can contribute to the development of emphysema associated aging in smokers. The Nrf2 SNP may be a predictive factor for smoking induced emphysema, and genotyping of Nrf2 SNP may serve as biomarker for emphysema prevention. 


\section{Introduction}

Chronic obstructive pulmonary disease (COPD) is a high ranking cause of death, and COPD risk assessment is an important part of primary prevention. Small airway lesions and emphysema contribute cooperatively to airflow limitations in COPD. Although emphysema can occur without the airflow limitation that defines COPD [1], pathological emphysema has been identified both in patients with COPD and in healthy adults with normal lung function. It has been demonstrated that emphysema is correlated with the development of airflow limitations in healthy adults, including those who have never smoked [2]. The most important environmental factor in development of emphysema is cigarettes smoke, which contain high concentrations of oxidants [3]. Oxidative stress promotes inflammatory gene expression through NF-kB signaling, which induces apoptosis of airway epithelial and vascular endothelial cells and leads to emphysema $[4,5]$.

NF-E2-related factor 2 (Nrf2) is a transcription factor and a master regulator of antioxidant responses to oxidative damage resulting from injury and inflammation. It has been reported that Nrf2-deficient mice are highly susceptible to cigarette smoke-induced emphysema [6]. Moreover, we have recently reported that expression of Nrf2 in bronchial and alveolar epithelial cells was significantly decreased in patients with COPD [7]. These studies indicate that there is a correlation between the Nrf2 regulated antioxidant defense system and oxidant-induced inflammation in the lungs. Some studies have reported that single nucleotide polymorphisms (SNPs) in Nrf2 contribute to annual decline in forced expiratory volume in one second (FEV1) in healthy smokers $[8,9]$. Because COPD in the Japanese population is predominantly emphysematous, and little is known about the role of Nrf2 SNPs on the pathology of emphysema, studying Nrf2 SNPs has the potential to provide insights into the nature of emphysema in Japanese patients and reveal new targets for therapies. Here, we focused on one Nrf2 SNP (rs6726395) shown to be related to a decline in lung 
function and conducted a study in the general Japanese population to evaluate correlations between this SNP and the extent of emphysema.

\section{Methods}

Study subjects

A total of 273 healthy subjects who underwent an annual health checkup at the MedCity 21 clinic of Osaka City

University Hospital between May 2017 and December 2017 were prospectively recruited into this study. All subjects

completed medical interviews, including medical history and cigarette smoking history (Pack year), physical examinations, routine blood studies, pulmonary function test (PFT), and high-resolution computed tomography (CT) of the chest. Based on data from PFT, 28 subjects were excluded from the study because they had already been diagnosed with COPD according to the Global Initiative for Chronic Obstructive Lung Disease (GOLD) guidelines [10]. A total of 245 subjects were enrolled for analysis. In this study, 'non-/light-smoker' was defined as a participant who smoked less than 10 Pack year, and 'smoker' was defined as a participant who smoked more than or equal to 10 Pack year. This study was approved by the ethics committee of Osaka City University Hospital (approval 3758), and all patients provided informed consent for their participation. All procedures were performed according to the guidelines of the Declaration of Helsinki.

The percentage of low-attenuation areas (\%LAA)

The chest CT was performed using a Whole Body X-ray CT System [Supria (16ch), Hitachi, Ltd., Tokyo, Japan)]. 
The scan time was 0.75 seconds, and the image matrix was $512 \times 512$ pixels. We reconstructed $1 \mathrm{~mm}$ thick thin-slice CT images for all lung fields, and all images were analyzed using Airway Inspector software (Surgical Planning Laboratory at Brigham and Women's Hospital, Boston, MA, USA). Areas with attenuation less than -950 Hounsfield units were defined as low-attenuation area (LAA), suggesting emphysema, and the ratio of LAA to the total area were defined as \%LAA. We quantified \%LAA for the upper-, middle-, lower-, and total lung areas, as previously described [11].

Single-Nucleotide Polymorphism (SNP) Selection and Genotyping

Genomic DNA was extracted from blood samples obtained from each participant using the Gentra Puregene Blood Kit Plus (Qiagen NV, Venlo, the Netherlands). SNP (rs6726395) genotyping in the Nrf2 gene was carried out for each participant with $50 \mathrm{ng}$ genomic DNA and the pre-designed TaqMan allele-specific polymerase chain reaction (PCR) assays using a GeneAmp PCR System (Applied Biosystems, Foster City, CA). All genotype (GG (major allele homozygotes), AG, and AA) of all subjects was detected.

\section{Statistics}

Correlations between \%LAA, clinical characteristics, and rs6726395 (G/A) genotypes in Nrf2 gene were examined.

Data are presented as mean \pm standard deviation (SD), unless otherwise stated. Statistical analyses were performed using JMP version 10.0.0 software for Windows (SAS Institute, Cary, NC, USA). In all statistical analyses, a p<0.05 was considered significant. We performed univariate analysis to evaluate the correlations of clinical characteristics 
with \%LAA or Nrf2 gene SNP (rs6726395). Fisher's exact test was used for comparisons of categorical variables such as gender, smoking status, and genotype. For continuous variables, such as age, body mass index (BMI), and PFT measurements, the correlation with \%LAA was assessed by Pearson's correlation coefficient analysis. For comparisons of categorical variables and continuous variables, parametrically unpaired Student's t-tests were used for comparison of two categorical group means. The correlations of rs6726395 genotypes with \%LAA were analyzed by multivariate linear regressions adjusted for potential confounding factors, such as age and BMI. We did not include other variables (gender and PFT measurements) as confounding factors in a consideration of multicollinearity.

\section{Results}

The characteristics of the 245 subjects without COPD [non-/light-smoker: $153(62.4 \%)$ and smoker: 92 (37.6\%)] in this study are summarized in Table 1. The comparison of \%LAA in clinical variables and each lung area is shown in Table 2 . The mean value of \%LAA in the total lung field of males was significantly higher than in females $(\mathrm{p}<0.001)$. As expected, the mean value of \%LAA in the total lung field of smokers was significantly higher than in non-/lightsmokers $(\mathrm{p}=0.021)$. The mean \%LAA in the upper lung field was significantly higher than in the lower lung field ( $\mathrm{p}<0.001)$. Dividing the subjects into smoker and non-/light-smoker groups produced similar results in both groups ( $<<0.001$; data not shown).

We performed univariate analyses to assess correlations between \%LAA and clinical variables in Table 3. The \%LAA showed significant but weak correlation with age in all subjects, including smokers and non-/lightsmokers $(\mathrm{r}=0.141, \mathrm{p}=0.028)$. There was no significant correlation between \%LAA and BMI. Regarding pulmonary 
function, \%LAA was significantly correlated with FVC $(r=0.427, p<0.001), \%$ FVC $(r=0.301, p<0.001)$, FEV1 $(\mathrm{r}=0.345, \mathrm{p}<0.001)$, and \% FEV1 $(\mathrm{r}=0.089, \mathrm{p}<0.001)$. However, there was no significant correlation between \%LAA and FEV1/FVC.

Genotype at SNP (rs6726395) in the Nrf2 gene was successfully detected for all subjects. The number of subjects with each genotype, GG, AG, and AA, was 116, 109, and 20, respectively. The group of genotype AA was very small, and was combined with group AG and designated group AG/AA. Relationships between the genotypes and clinical variables are shown in Table 4. There were no significant differences between any of the measured variables (age, gender, BMI, smoking status, the parameters of PFT, and \%LAA) and genotypes (GG and AG/AA). Although the difference did not reach the statistical significance, the \%LAA of genotype AG/AA was higher than that of genotype GG.

As emphysema in the upper lung field was prominent, the \%LAA of the upper lung field in smokers was analyzed. Multivariate linear regressions of correlations between Nrf2 gene SNP genotypes (rs6726395) and \%LAA of the upper lung field in smokers are shown in Table 5. In univariate analysis, the \%LAA in the upper lung field showed a significant correlation with age $(\mathrm{r}=0.333, \mathrm{p}=0.022)$ and $\mathrm{BMI}(\mathrm{r}=-0.298, \mathrm{p}=0.042)$ in smokers without COPD with genotype GG (Figure $1 \mathrm{~A}, \mathrm{~B}$ ). While, the \%LAA in the upper lung field showed no correlation with age and BMI in smokers without COPD with genotype AG/AA (Figure 1 C,D). Furthermore, adjusted for potential confounding factors as BMI, \%LAA in the upper lung field was significantly correlated with age in smokers with genotype GG $(\mathrm{p}=0.0497)$, while the \%LAA was not significantly correlated with age in smokers with genotype AG/AA $(\mathrm{p}=0.582)$. These correlations were not observed in non-/light-smokers with either genotype. 


\section{Discussion}

In the present study, we determined that a polymorphism of Nrf2 gene accelerated progress of upper lung emphysema associated aging in smokers without COPD. We showed a correlation between a SNP of the Nrf2 gene and the extent of emphysema associated aging in the general Japanese population, and our results indicate the presence of a gene-environment interaction in \%LAA of the upper lung field. Several studies have implicated Nrf2 SNPs in some pulmonary diseases, such as lung adenocarcinoma, acute lung injury, and bronchial asthma [12-14]. Moreover, in some studies of healthy individuals from the general population, Nrf2 SNPs were shown to be correlated with FEV1 $[8,9]$. We therefore hypothesized that Nrf2 SNPs could play some role in the development of emphysema associated aging due to cigarette smoking. To investigate this, we evaluated correlations between a Nrf2 SNP and the extent of emphysema associated aging in the general Japanese population.

Cigarette smoke contains various toxic substances, including high concentrations of oxidants [3]. Oxidants induce local inflammation, which develops apoptosis in airway epithelial and vascular endothelial cells and leads to emphysema [4, 5]. For this reason, the lungs of smokers easily become emphysematous. In the present study, the mean \%LAA in smokers was significantly higher than in non-/light-smokers. Emphysema is generally found in the upper lung field in COPD patients [11, 15], and it has been suggested that the cause of this distributional heterogeneity is the ventilation-perfusion mismatch between the upper and lower lobes [16]. In the current study the mean \%LAA in upper lung field was higher than that in lower lung field, even in smokers without COPD.

The initiation and development of emphysema can be impacted aging of the lung [17]. It has also been reported that 
cellular senescence associated aging promotes abnormal cell turnover and development of emphysema [18]. We have also previously reported that levels of soluble $\alpha$-klotho, identified as a putative aging-suppressor gene, were significantly lower in smokers with COPD [19]. Therefore, aging is also a risk factor for emphysema, and the extent of emphysema was found to be weakly correlated with age in the current study.

Although recent large-scale genome-wide association studies have identified several loci related to measures of pulmonary function [20, 21], SNPs related to Nrf2 gene were not listed as whole subjects in these reports. Based on a report about the correlation between Nrf2 SNPs and FEV1 which focused on Japanese people with COPD, we have selected SNP (rs6726395) with relatively high minor allele frequency as the target SNP in this study [8]. The frequency of this allele differs by race, and the frequency of allele A is low in Asians [22]. The rs6726395 SNP is located in the first intron of the Nrf2 gene, and the specific functions of this SNP are unclear. In our study, the \%LAA of the upper lung field was significantly correlated with age in smokers with genotype GG but not in smokers with genotype AG/AA. In major allele homozygotes $(\mathrm{GG})$, our results reflect the general concept that cigarette smoke accelerates development of upper lung emphysema associated aging. By contrast, in genotype AG/AA with minor allele (A), this correlation was not observed. The rs6726395 SNP with allele A have been reported to have lower expression of Nrf2 compared with the allele G [23, 24], and genotype AG/AA might susceptible to oxidant induced lung damage, i.e. emphysema. Interestingly, \%LAA in genotype AG/AA was higher than in genotype GG with marginal significance. This tendency supports the reports of the lower expression of Nrf2 in allele A. In general, about $20 \%$ of smokers develop clinical COPD [25], but it is unclear what fraction of smokers develop smoking-induced emphysema associated aging. In the current study, genotyping showed that $52.7 \%$ (AA/AG) of the 
study population was sensitive to smoking-induced emphysema. COPD is thought to have multiple genetic and environmental factors related to pathogenesis, which might explain this discrepancy.

PFT is recommended for COPD diagnosis [10] and early screening for unattributed or potential COPD subjects identified in health check-ups. PFT for COPD screening is comparatively easy and less invasive than other types of testing because it does not require irradiation. Recently low-dose chest CT has proved to be an efficient method for lung cancer screening [26]. Although the settings of chest CT and reconstruction algorithms need to be adjusted, chest CT screening at health check-ups can be conducted for both lung cancer and emphysema screening. Genotyping of the Nrf2 gene is less invasive than PFT or chest CT, because only sampling of genomic DNA is necessary for such screening. Moreover, genotyping can assess future risk of emphysema/COPD, while PFT and chest CT can only assess the lung at the present time.

Prevention of diseases can be achieved primarily through education and secondarily through early detection. Recently, earlier prevention has been advocated because environmental adjustment is important for public health. Incidence of inflammatory lung diseases like COPD is increased by noxious gases, mainly cigarette smoking. In addition to cigarette smoking, the issues of environmental pollution from second-hand smoke or air pollution must be considered as contributors to inflammatory lung diseases. Since genotyping can be used to determine susceptibility, it may be a tool that can be used in early prevention efforts. Results from the current study can contribute to development of this prevention concept.

Our study population had a limited sample size and all subjects were enrolled from single center. As a result of this sample size limitation we identified only a small number of smokers with genotype AA, and so this group was 
combined with the genotype AG group. For the same reason, we have not assessed other SNPs in the Nrf2 gene. Also, the functional effect of the Nrf2 SNP (rs6726395) in this study is still unknown. However, in the present study, we showed correlations between a SNP (rs6726395) and the extent of upper lung field emphysema associated aging in Japanese smokers. This might contribute to personalized medicine or prevention from COPD development, and Nrf2 could be an intervention target for COPD prevention and treatment. Further studies using larger populations will be required to confirm our findings.

\section{Conclusion}

A polymorphism rs6726395 in Nrf2 can contribute to the development of emphysema associated aging in smokers. The Nrf2 SNP may be a predictive factor for smoking induced emphysema, and genotyping of Nrf2 SNP may be serve as biomarker for emphysema prevention.

\section{Acknowledgments}

Genotyping analysis were performed in the Research Support Platform of Osaka City University Graduate School of Medicine.

\section{Conflict of Interest}

The authors have stated explicitly that there is no conflict of interest in connection with this article. 


\section{Figure Legend}

Fig.1 Correlation between \%LAA in upper lung field and age or BMI in smokers without COPD with each genotype (GG and AG/AA)

A: \%LAA in upper lung field showed a significant correlation with age $(r=0.333, p=0.022)$ in smokers without COPD with genotype GG

B: \%LAA in upper lung field showed a significant correlation with BMI $(r=-0.298, p=0.042)$ in smokers without COPD with genotype GG

C: \%LAA in upper lung field showed no correlation with age $(r=0.099, p=0.519)$ in smokers without COPD with genotype AG/AA

D: \%LAA in upper lung field showed no correlation with BMI $(\mathrm{r}=-0.293, \mathrm{p}=0.051)$ in smokers without COPD with genotype AG/AA 


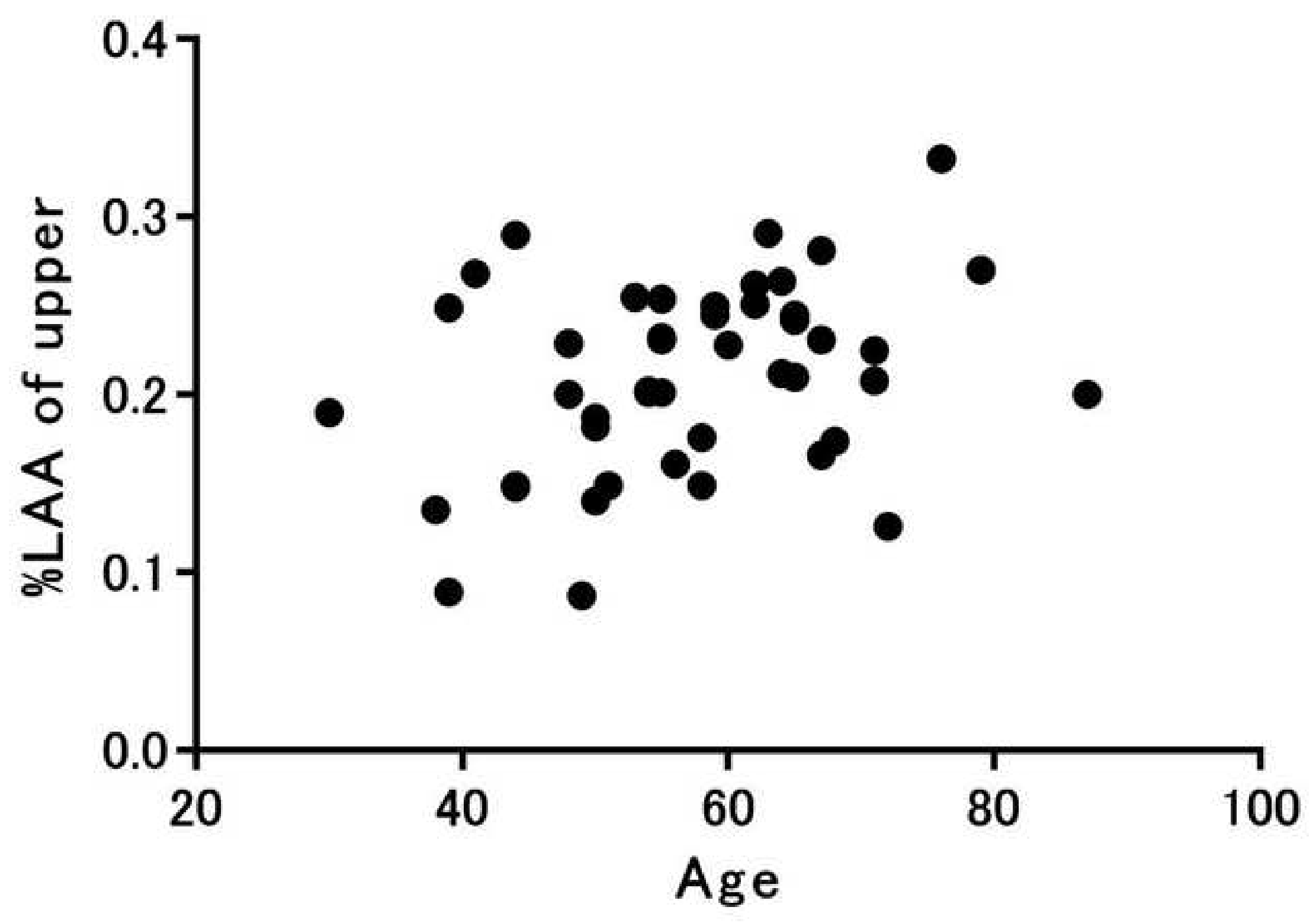




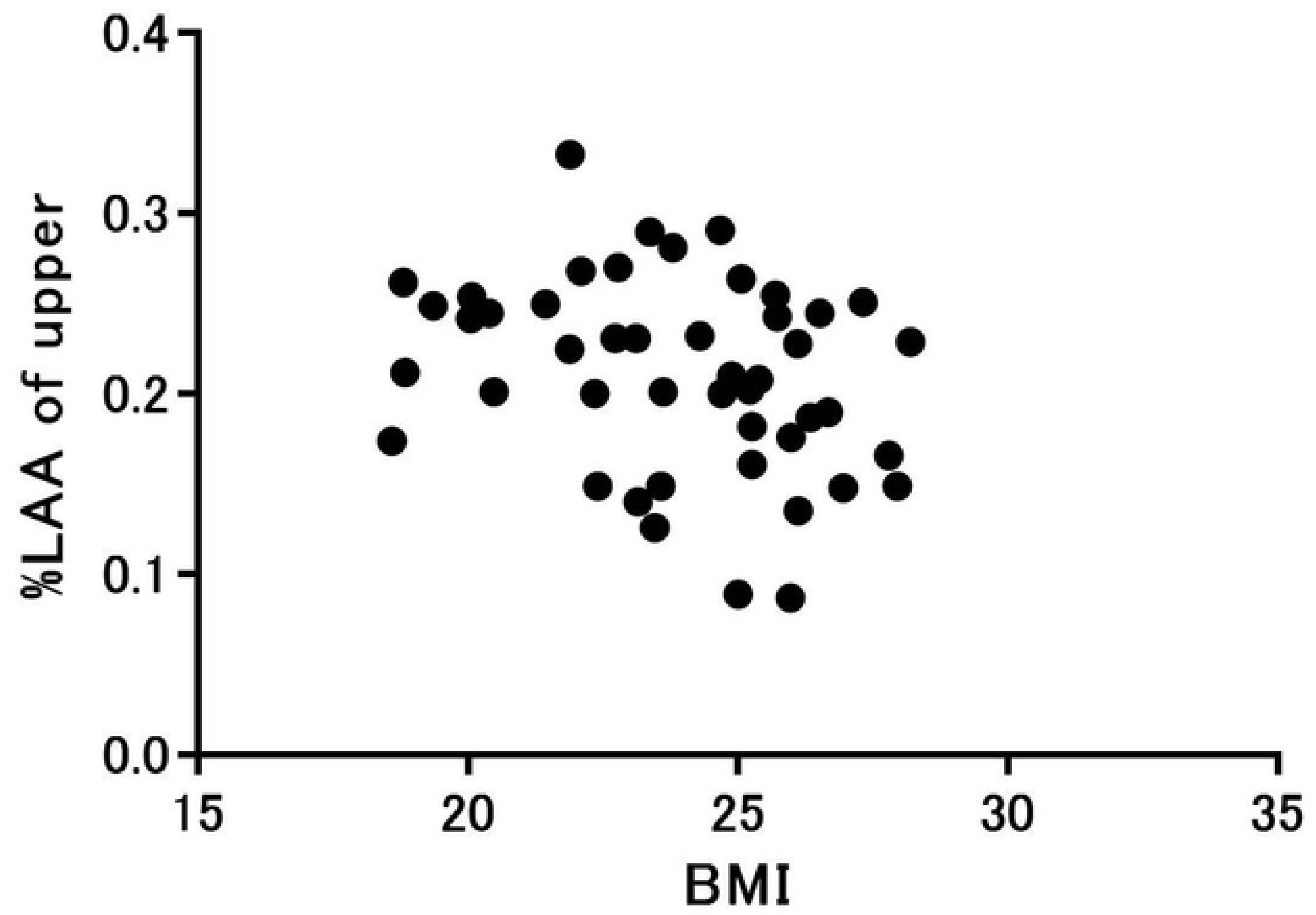




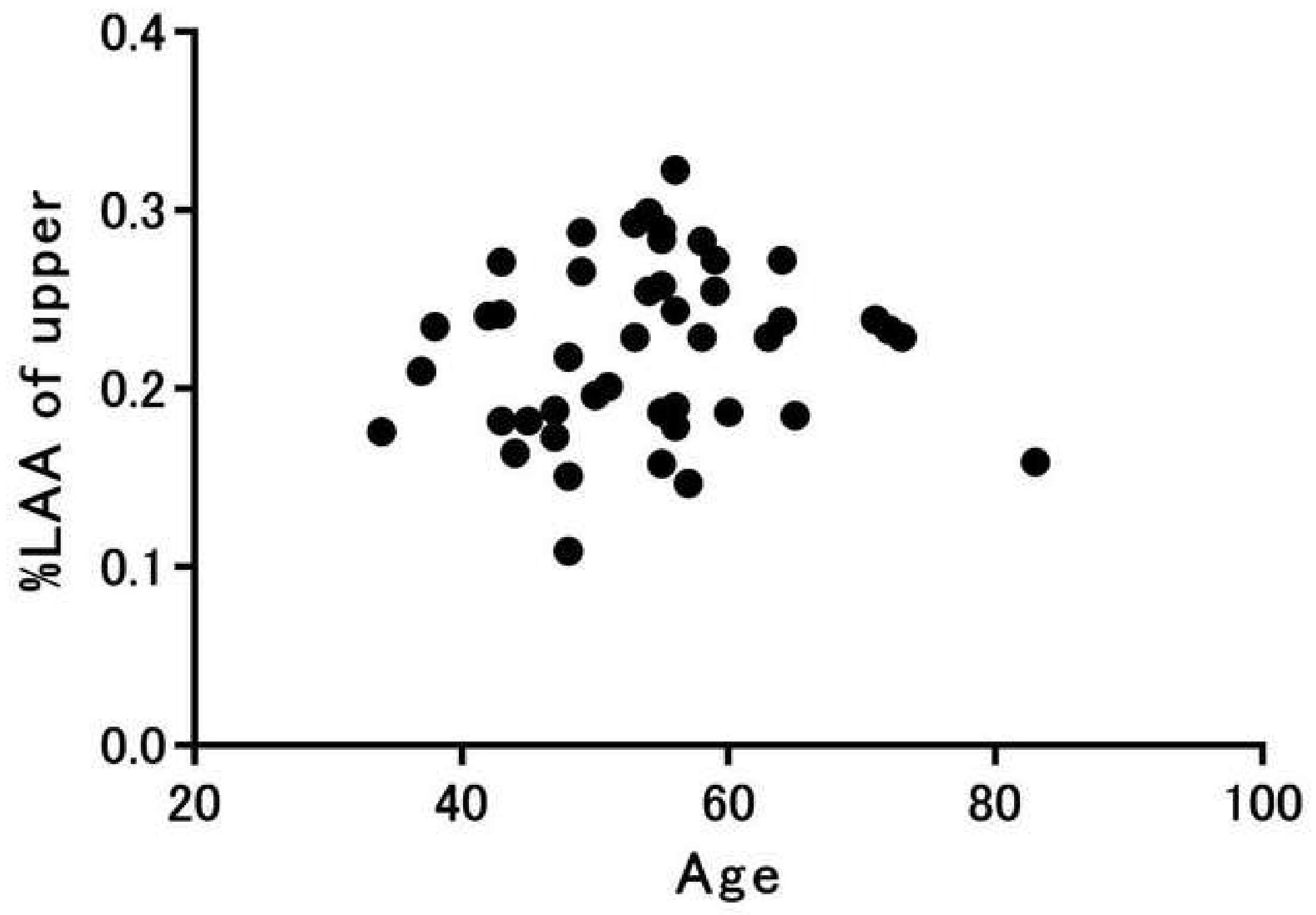




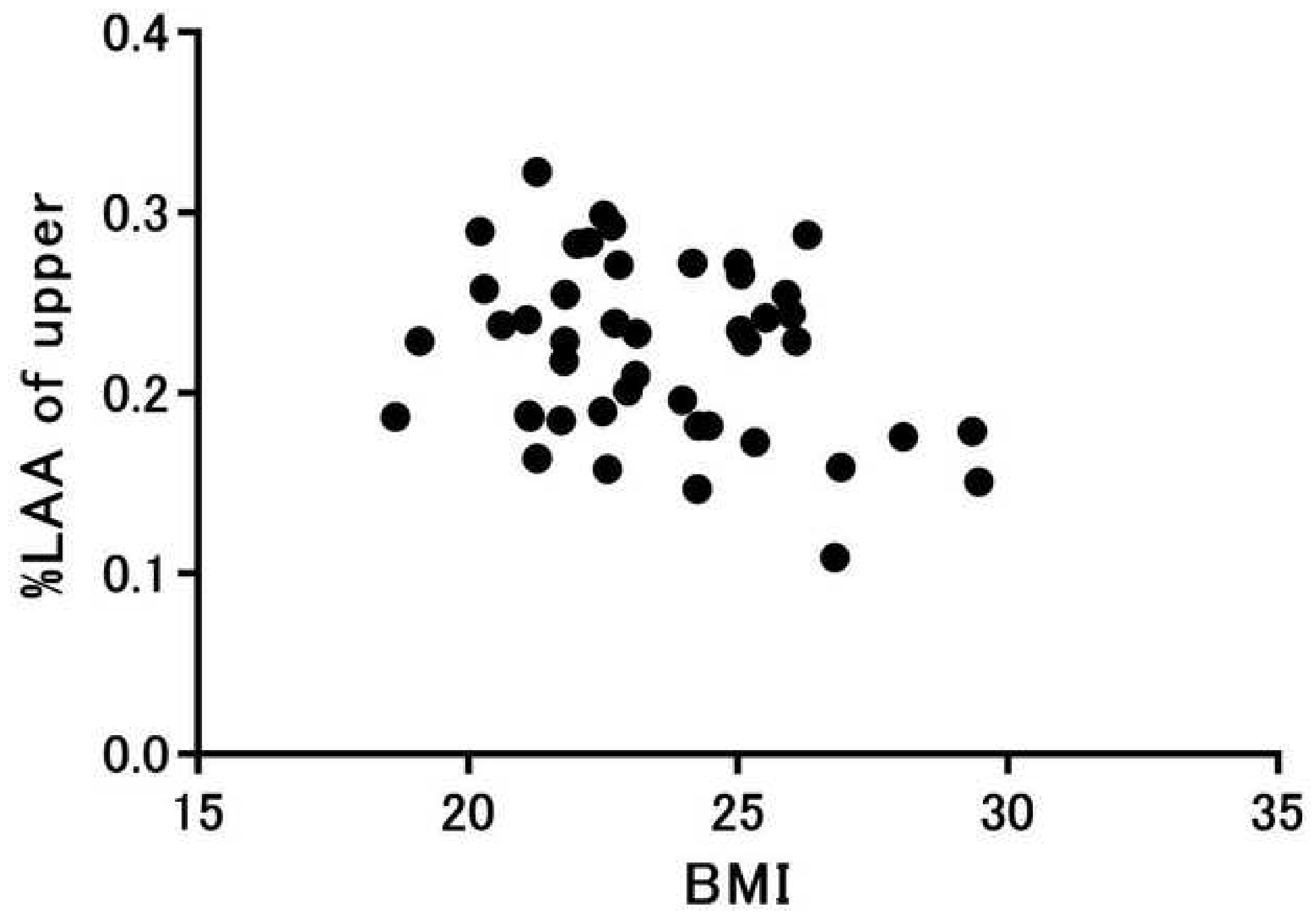


Table 1 Characteristics of the study subjects

\begin{tabular}{|c|c|}
\hline Characteristics & Subjects $(n=245)$ \\
\hline Age (years) & $53.6 \pm 11.7$ \\
\hline \multicolumn{2}{|l|}{ Gender - n (\%) } \\
\hline Male & $150(61.2)$ \\
\hline Female & $95(38.8)$ \\
\hline BMI (kg/m2) & $22.9 \pm 2.7$ \\
\hline FVC (L) & $3.70 \pm 0.85$ \\
\hline FVC \% predicted (\%) & $102.9 \pm 12.7$ \\
\hline $\mathrm{FEV}_{1}(\mathrm{~L})$ & $2.93 \pm 0.67$ \\
\hline $\mathrm{FEV}_{1} / \mathrm{FVC}(\%)$ & $79.6 \pm 5.11$ \\
\hline $\mathrm{FEV}_{1} \%$ predicted (\%) & $97.8 \pm 12.4$ \\
\hline \multicolumn{2}{|l|}{ Pack year - n(\%) } \\
\hline$<10$ & $153(62.4)$ \\
\hline $10 \leqq$ & $92(37.6)$ \\
\hline
\end{tabular}

Data are presented as mean \pm SD or $n(\%)$ 
Table 2 Comparing of \%LAA in clinical variables and each lung areas

\begin{tabular}{lll}
\hline Variables & $\%$ LAA * (mean $\pm S D)$ & p value \\
\hline Gender & $21.2 \pm 5.3$ & $<0.001$ \\
Male & $16.6 \pm 4.5$ & \\
Female & & 0.021 \\
Pack year & $18.8 \pm 5.4$ & \\
$<10$ & $20.5 \pm 5.4$ & \\
$10 \leqq$ & & \\
Lung area & $20.6 \pm 5.2 * *$ & \\
upper & $19.5 \pm 5.5$ & \\
middle & $18.2 \pm 6.2 * *$ & \\
lower & $19.4 \pm 5.5$ & \\
total &
\end{tabular}

*\%LAA : the ratio of low-attenuation areas to all lung areas

$* * p<0.001$ (upper vs lower) 
Table 3 Correlation between \%LAA and clinical variables

\begin{tabular}{lll}
\hline Variables & correlation coeffcient & p value \\
\hline Age & 0.141 & 0.028 \\
BMI (kg/m2) & -0.004 & n.s. \\
FVC (L) & 0.427 & $<0.001$ \\
FVC \% predicted (\%) & 0.301 & $<0.001$ \\
FEV $_{1}(\mathrm{~L})$ & 0.345 & $<0.001$ \\
$\mathrm{FEV}_{1} \%$ predicted (\%) & 0.089 & $<0.001$ \\
$\mathrm{FEV}_{1} / \mathrm{FVC}(\%)$ & -0.286 & n.s. \\
\hline
\end{tabular}


Table 4 Classification of clinical variables with respect to rs6726395 genotypes

\begin{tabular}{|c|c|c|c|}
\hline \multirow[t]{2}{*}{ Variables } & \multicolumn{2}{|c|}{ Nrf2 gene SNP (rs6726395) } & \multirow[t]{2}{*}{$p$ value } \\
\hline & GG & $\mathrm{AA} / \mathrm{AG}$ & \\
\hline Subjects & $116(47.3)$ & $129(52.7)$ & \\
\hline Age & $53.5 \pm 11.7$ & $53.7 \pm 11.8$ & n.s. \\
\hline Gender - n(\%) & & & n.s. \\
\hline Male & $73(48.7)$ & $77(51.3)$ & \\
\hline Female & $43(45.3)$ & $52(54.7)$ & \\
\hline $\mathrm{BMI}\left(\mathrm{kg} / \mathrm{m}^{2}\right)$ & $23.1 \pm 2.7$ & $22.7 \pm 2.7$ & n.s. \\
\hline Pack year - n(\%) & & & n.s. \\
\hline$<10$ & $69(45.1)$ & $84(54.9)$ & \\
\hline $10 \leqq$ & $47(51.1)$ & 45 (48.9) & \\
\hline FVC (L) & $3.72 \pm 0.85$ & $3.67 \pm 0.84$ & n.s. \\
\hline FVC \% predicted (\%) & $102.4 \pm 12.8$ & $103.4 \pm 12.7$ & n.s. \\
\hline $\mathrm{FEV}_{1}(\mathrm{~L})$ & $2.96 \pm 0.68$ & $2.92 \pm 0.65$ & n.s. \\
\hline $\mathrm{FEV}_{1} / \mathrm{FVC}(\%)$ & $79.5 \pm 5.2$ & $79.7 \pm 5.0$ & n.s. \\
\hline $\mathrm{FEV}_{1} \%$ predicted (\%) & $97.0 \pm 12.0$ & $98.4 \pm 12.7$ & n.s. \\
\hline \%LAA (upper) & $20.1 \pm 5.3$ & $20.9 \pm 5.1$ & n.s. \\
\hline \%LAA (middle) & $19.0 \pm 5.7$ & $20.0 \pm 5.3$ & n.s. \\
\hline \%LAA (lower) & $17.6 \pm 6.5$ & $18.7 \pm 6.0$ & n.s. \\
\hline \%LAA (total) & $18.9 \pm 5.6$ & $19.9 \pm 5.3$ & n.s. \\
\hline
\end{tabular}

Data was expressed mean \pm SD otherwise indicated. 
Table 5 Multivariate linear regressions for correlation between age or BMI in each genotype at rs6726395 and \%LAA of upper lung area in smokers

\begin{tabular}{lllll}
\hline Genotype & Variable & Correlation coeffcient & $p$ value & Corrected $p$ value \\
\hline GG & Age & 0.333 & 0.022 & $<0.05(0.0497)$ \\
& BMI & -0.298 & 0.042 & n.s. \\
AA/AG & Age & 0.099 & n.s. & n.s. \\
& BMI & -0.293 & n.s. & n.s. \\
\hline
\end{tabular}




\section{References}

[1] O. Auerbach, E.C. Hammond, L. Garfinkel et al (1972) Relation of smoking and age to emphysema. Whole-lung section study, N Engl J Med 286(16) 853-7.

[2] P.A. Gevenois, P. De Vuyst, V. de Maertelaer et al (1996) Comparison of computed density and microscopic morphometry in pulmonary emphysema, Am J Respir Crit Care Med 154(1) 187-92.

[3] D.F. Church, W.A. Pryor (1985) Free-radical chemistry of cigarette smoke and its toxicological implications, Environ Health Perspect 64:111-26.

[4] K. Aoshiba, N. Yokohori, A. Nagai (2003) Alveolar wall apoptosis causes lung destruction and emphysematous changes, Am J Respir Cell Mol Biol 28(5) 555-62.

[5] W.A. Pryor, M.M. Dooley, D.F. Church (1985) Mechanisms of cigarette smoke toxicity: the inactivation of human alpha-1-proteinase inhibitor by nitric oxide/isoprene mixtures in air, Chem Biol Interact 54(2) 171-83.

[6] T. Iizuka, Y. Ishii, K. Itoh et al (2005) Nrf2-deficient mice are highly susceptible to cigarette smokeinduced emphysema, Genes Cells 10(12) 1113-25.

[7] K. Yamada, K. Asai, F. Nagayasu et al (2016) Impaired nuclear factor erythroid 2-related factor 2 expression increases apoptosis of airway epithelial cells in patients with chronic obstructive pulmonary disease due to cigarette smoking, BMC Pulm Med 16:27.

[8] H. Masuko, T. Sakamoto, Y. Kaneko et al (2011) An interaction between Nrf2 polymorphisms and smoking status affects annual decline in FEV1: a longitudinal retrospective cohort study, BMC Med Genet 
12:97.

[9] M. Siedlinski, D.S. Postma, J.M. Boer et al (2009) Level and course of FEV1 in relation to polymorphisms in NFE2L2 and KEAP1 in the general population, Respir Res 10:73.

[10] J. Vestbo, S.S. Hurd, A.G. Agusti et al (2013) Global strategy for the diagnosis, management, and prevention of chronic obstructive pulmonary disease: GOLD executive summary, Am J Respir Crit Care Med 187(4):347-65.

[11] M. Xie, W. Wang, S. Dou et al (2016) Quantitative computed tomography measurements of emphysema for diagnosing asthma-chronic obstructive pulmonary disease overlap syndrome, Int J Chron Obstruct Pulmon Dis 11:953-61.

[12] Y. Okano, U. Nezu, Y. Enokida et al (2013) SNP (-617C>A) in ARE-like loci of the NRF2 gene: a new biomarker for prognosis of lung adenocarcinoma in Japanese non-smoking women, PLoS One 8(9) e73794. [13] D.S. O'Mahony, B.J. Glavan, T.D. Holden et al (2012) Inflammation and immune-related candidate gene associations with acute lung injury susceptibility and severity: a validation study, PLoS One 7(12) e51104.

[14] I. Ungvari, E. Hadadi, V. Virag et al (2012) Relationship between air pollution, NFE2L2 gene polymorphisms and childhood asthma in a Hungarian population, J Community Genet 3(1) 25-33.

[15] H.N. Bastos, I. Neves, M. Redondo et al (2015) Influence of emphysema distribution on pulmonary function parameters in COPD patients, J Bras Pneumol 41(6) 489-95.

[16] D.W. Cockcroft, S.L. Horne (1982) Localization of emphysema within the lung. An hypothesis based 
upon ventilation/perfusion relationships, Chest 82(4) 483-7.

[17] R.A. Maciewicz, D. Warburton, S.I. Rennard (2009) Can increased understanding of the role of lung development and aging drive new advances in chronic obstructive pulmonary disease?, Proc Am Thorac Soc 6(7) 614-7.

[18] T. Tsuji, K. Aoshiba, A. Nagai (2006) Alveolar cell senescence in patients with pulmonary emphysema, Am J Respir Crit Care Med 174(8) 886-93.

[19] Y. Kureya, H. Kanazawa, N. Ijiri et al (2016) Down-Regulation of Soluble alpha-Klotho is Associated with Reduction in Serum Irisin Levels in Chronic Obstructive Pulmonary Disease, Lung 194(3) 345-51. [20] D.B. Hancock, M. Eijgelsheim, J.B. Wilk et al (2010) Meta-analyses of genome-wide association studies identify multiple loci associated with pulmonary function, Nat Genet 42(1) 45-52.

[21] E. Repapi, I. Sayers, L.V. Wain et al (2010) Genome-wide association study identifies five loci associated with lung function, Nat Genet 42(1) 36-44.

[22] https://www.ncbi.nlm.nih.gov/snp/rs6726395\#frequency_tab.

[23] T. Khunluck, V. Kukongviriyapan, A. Puapairoj et al (2014) Association of NRF2 polymorphism with cholangiocarcinoma prognosis in Thai patients, Asian Pac J Cancer Prev 15(1) 299-304.

[24] E. Synowiec, T. Sliwinski, K. Danisz et al (2013) Association between polymorphism of the NQO1, NOS3 and NFE2L2 genes and AMD, Front Biosci (Landmark Ed) 18:80-90.

[25] Cigarette smoking and health. American Thoracic Society, Am J Respir Crit Care Med 153(2) (1996) 861-5. 
[26] J.E. Walter, M.A. Heuvelmans, G.H. Bock et al (2018) Characteristics of new solid nodules detected in incidence screening rounds of low-dose CT lung cancer screening: the NELSON study, Thorax 73(8) 741-747. 